\title{
An Analysis of Peak Demand Reductions Due to Elasticity of Domestic Appliances
}

\author{
P. Srikantha, C. Rosenberg \\ Dept. of Electrical and Computer Engineering \\ University of Waterloo \\ Waterloo, Ontario N2L 3G1 Canada \\ \{psrikant,cath\}@uwaterloo.ca
}

\author{
S. Keshav \\ David R. Cheriton School of Computer Science \\ University of Waterloo \\ Waterloo, Ontario N2L 3G1 Canada \\ keshav@uwaterloo.ca
}

\begin{abstract}
Unlike prior work on demand management, which typically requires industrial loads to be turned off during peak times, this paper studies the potential to carry out demand response by modifying the elastic load components of common household appliances. Such a component can decrease its instantaneous power draw at the expense of increasing its duration of operation with no impact on the appliance's lifetime. We identify the elastic components of ten common household appliances. Assuming separate control of an appliance's elastic component, we quantify the relationship between the potential reduction in aggregate peak and the duration required to complete the operation of appliances in four geographic regions: Ontario, Quebec, France and India. We find that even with a small extension to the operation duration of appliances, peak demand can be significantly reduced in all four regions both during winter and summer. For example, during winter in Quebec, a nearly $125 \mathrm{MW}$ reduction in peak demand can be obtained with just a $10 \%$ increase in appliance operation duration. We conclude that exploiting appliance elasticity to reduce peak power demand should be an important consideration for appliance manufacturers. From a policy perspective, our study gives regulators the ability to quantitatively assess the impact of requiring manufacturers to conform to "smart appliance" standards.
\end{abstract}

\section{Categories and Subject Descriptors}

I.6 [Simulation and Modeling]: Model Validation and Analysis

\section{General Terms}

Design, Measurement, Verification

\section{Keywords}

Electrical Grid, Load Modeling

Permission to make digital or hard copies of all or part of this work for personal or classroom use is granted without fee provided that copies are not made or distributed for profit or commercial advantage and that copies bear this notice and the full citation on the first page. To copy otherwise, to republish, to post on servers or to redistribute to lists, requires prior specific permission and/or a fee.

e-Energy 2012, May 9-11 2012, Madrid, Spain.

Copyright 2012 ACM 978-1-4503-1055-0/12/05 ...\$10.00.

\section{INTRODUCTION}

The generation and transmission infrastructure in the power grid is provisioned at a great cost for rarely-occurring demand peaks. For example, in 2006, about $7 \%$ of Ontario's generation capacity was idle $99.6 \%$ of the time. The Ontario Power Authority estimates that a reduction of peak electricity demand by 7,100 MW on a base of 27,000 MW could result in savings of up to $\$ 27$ billion over 20 years [18].

This problem is well understood: utilities already implement demand response schemes to shave peak demand from industrial customers. However, demands from the residential sector in regions such as Ontario, Quebec, France and India represent about one third of overall demand in each region $[23,1,6,8]$. Moreover, it is expected that the demand from the residential sector will continue to rise. In United States, for example, it is projected that residential electricity consumption will increase by an additional $68 \%$ from 2003 to 2025 [10]. Therefore, there is an urgent need to study demand reduction in the residential sector. Residential electricity demand primarily arises from a handful of power-hungry appliances. Most such appliances have an elastic load component that can decrease its instantaneous power draw, at the expense of increasing its duration of operation, with no impact on the appliance's lifetime. Examples include resistive heating elements and energy storage components. Assuming separate control of elastic components of an appliance, decreasing the load when elastic components are active can reduce demand peaks.

Although this idea is simple, quantifying the potential gains from appliance elasticity, necessary to determine if modifying appliances is worth the effort, is complex. This is because the gain depends on the appliance mix in a particular region, the number of elastic phases in these appliances, the amount of power consumed during elastic phases, the usage patterns of these appliances, and the level of consumer tolerance to the extension of the operation durations of appliances. To rein in this complexity, we focus our attention on four regions: Ontario, Quebec, France and India. We gather a comprehensive data set on the common appliances, penetration rates, load profiles, and usage patterns for these regions. This allows us to use simulations to estimate the reduction in the peak demand as a function of the operation duration extension factor in each of the four regions.

We find that even with a small extension to the operation duration of appliances, peak demand can be significantly reduced in all four regions both during winter and summer. For example, we find that during winter in Quebec (when 
the peak typically occurs), there can be a nearly $125 \mathrm{MW}$ reduction in peak demand with just a $10 \%$ increase in the duration of operation of household appliances. Interestingly, during summer, this reduction is over and above that can be obtained by careful scheduling of residential air conditioners [26].

Our results have practical implications. Appliance manufacturers typically focus on energy efficiency rather than peak-power reduction. We show that reducing power consumption peaks is both feasible and effective. From a policy perspective, our study gives regulators the ability to assess the impact of requiring manufacturers to reduce peak power and build appliances that provide separate control of elastic load components.

Our main contributions are that we have:

- Identified the elasticity property of appliances that allows the reduction of the instantaneous power drawn with a quantifiable impact on the appliance operation duration and no impact on their lifetime.

- Provided a detailed taxonomy of the ten most common household appliances with respect to their elasticity.

- Collected, analyzed and compared a comprehensive dataset on the types of appliances used, penetration rates, load profiles of appliances and usage patterns for Ontario, Quebec, France and India.

- Quantified the impact on peak power demand reductions for Ontario, Quebec, France and India as a function of the appliance operation duration extension factor via simulations.

- Validated the simulations through detailed probabilistic computations.

The remainder of this paper is organized as follows. An indepth overview of appliances is presented in Section 2. The assumptions underlying our work are explicitly presented in Section 3. We present in Section 4 the method used to shape appliance power usage to best take advantage of the extension in the duration of operation of elastic components. Section 5 presents evaluation methodology and the data sets used in our work. The results of our work quantifying the range of peak demand reductions that can be expected for Ontario, Quebec, India and France are presented in Section 6. We discuss related work in Section 7 and the paper is concluded in Section 8.

\section{APPLIANCES - A TAXONOMY}

In this section, we present an in-depth view of the inner workings of typical household appliances. We first define the four main classes of components that can be present in appliances. Then we examine ten common household appliances and identify their elastic components.

\subsection{Components of Appliances}

There are four main classes of components present in today's appliances [25]. These are purely resistive elements, motors, electronic devices and energy storage systems.

Purely resistive components contain a heating element that generates heat directly proportional to the amount of electrical energy passed through the coils. These types of components are very tolerant to variations in power and frequency. The heating elements can only be damaged due to overheating and wear if exposed to very high power. Otherwise, the heat or light output is reduced. For example, lights will be dimmed at lower power and heater will produce less heat. Importantly, there is no impact on the lifetime of purely resistive components when the power drawn is reduced.

A motor, in simplistic terms, is a generator operating backwards. It is present in virtually any appliance that converts electrical energy to mechanical energy. The most common motor type found in today's households is an induction motor. Unlike purely resistive loads, motors are sensitive to fluctuations in power. Excessive heating of the coils caused by these fluctuations can result in the degradation of the motor. This can be worsened due to the harmonic content introduced in the motors by these imbalances. Sudden changes in power can also damage the motor controls.

Electronic Devices operate on low voltage Direct Current (DC). Purely electronic devices require a small current to operate because they do no significant physical work. The sensitivity of these devices to power quality depends on the type of loads the electronic devices are coupled with. A simple example is an LED clock that resets due to sudden power transitions. Reducing these devices' power consumption is usually not necessary since they consume little power.

An example of a device containing energy storage is a Plug-in Hybrid Electric Vehicle (PHEV). These devices discharge their batteries during active periods. When connected to the grid, the batteries can replenish their energy. The charging can be done at different power levels without damaging the batteries [20].

In our approach, we wish to modify the power consumption of appliances when necessary. However, as outlined in this section, certain components in appliances are sensitive to variations in power supplied. It is clear that only components falling under the purely resistive and energy storage classes will experience no lifetime or reliability impact from such variations. We will refer to a period having such a component active to be an elastic phase.

\subsection{Identification of Elastic Phases in Appli- ances}

We now identify the common household appliances that are major consumers of power, examine their operating cycles and identify the elastic phases in these cycles, if any.

The internal workings of appliances can differ according to the region. For example, dishwashers can use a local heating element to heat water or can directly draw hot water from a central water heater which may use gas for heating. Typically, the type of appliance used in a region is uniform. The Smart-A project has identified the following ten appliances as common domestic loads: washing machine, tumble dryer, dishwasher, oven, stove, refrigerator, freezer, air conditioner, water heater, and space heater [24]. Although PHEVs will become an integral part of a household in the near future [13], we do not include it in our study because it does not have a noticeable penetration in today's households. We next provide an overview of the various components present in these appliances.

A washing machine (WM) consists of a tub, drum, and either a heating element and water pipes or hot and cold water pipes. The drum is spun at high speeds with an in- 
duction motor (an inelastic component) for the rinse and wash cycles. Some washing machines use a local heating element (elastic component) to heat water to temperatures depending on the cycle selected by the consumer. The heating element typically consumes $2000 \mathrm{~W}$ when active and is the most power demanding component [24]. Elastic phases occur whenever the heating element is active. The other washing machines mix hot water obtained from a central water heating system with cold water to achieve the desired temperatures. These washing machines do not have any local elastic components and the average power consumed is around 500W during operation [2].

A tumble dryer (TD) consists of a drum and a purely resistive heating element. The drum is spun with an induction motor which consumes low power. The heating element is used to heat up the air. This hot air is passed over the wet laundry and the humid air is passed out into the ambient environment. The heating element which is the only elastic component in the dryer uses the highest power. For example, a typical dryer in France has one elastic phase during which the heating element consumes $2500 \mathrm{~W}$ for about 45 minutes [24]. Since the drum and the heating element work together, if the time taken to complete the drying is extended because the power consumed by the heating element is reduced, the drum can continue to spin for the extended time without significant impact as its power consumption is a fraction of that required for the heating element.

A dishwasher $(D W)$ consists of a rotor, a local heating element and connection to water pipes (optionally connection to hot and cold water pipes). Some dishwashers use a local heating element to heat water. Others mix hot water drawn from a central water heating system with cold water to attain desired temperatures. The rotor, operated by an induction motor, sprays this water on the dishes. At the end of a wash cycle, dishes are heated by the heating element to very high temperatures to aid with the drying process. Elastic phases occur whenever the heating element is active. The heating element typically draws about 2000W [24].

An oven $(O)$ and a stove $(S)$ consist of a range which is a purely resistive heating element that switches on and off to maintain the heat specified by the consumer. The heating element consumes about $1500 \mathrm{~W}$ in a stove and $600 \mathrm{~W}$ in an oven for about half of the cycles' duration [24]. This is also the maximum power consumed during the entire cooking process operating cycle. Elastic phases in the stove and oven occur whenever the heating element is active.

A refrigerator $(R F)$, a freezer $(F)$, and an air conditioner (AC) consist of a compressor operated with an induction motor that compresses heated refrigerant gas. These appliances have thermal inertia and their operation relies on external temperatures and internal temperature setpoints. The compressor needs to be on for at least 10 minutes to allow proper compression of gas. It cannot be on for too long either as the heat produced due to compression can damage the equipment. The compressor requires to be switched off for a period to allow equalization of pressure. Due to the sensitivity of the components present in these appliances to power variations, we classify these appliances as having no significant elastic component. ACs can be major contributors to peak load. An AC can consume more than $1700 \mathrm{~W}$ in each cycle [24]. The power consumption of RF and $F$ is around $140 \mathrm{~W}$ each and is not as high as that of an AC [24].

An electric water heater $(E W H)$ and an electric space

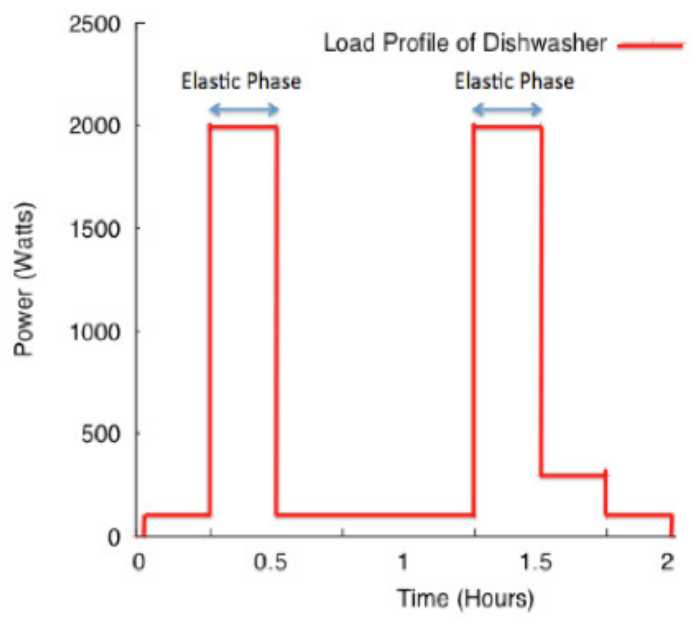

Figure 1: Load profile of a dishwasher with a local heating element for heating water.

heater (ESH) consist of a purely resistive heating element. The heating element consumes about $2000 \mathrm{~W}$ to $6000 \mathrm{~W}$ for the water heater and $8500 \mathrm{~W}$ for the space heater [24]. The actual power consumed depends on the type of heaters - centralized or standby. For heaters with storage, less power is required. Elastic phases in both appliances occur whenever the heating elements are active. The electric water heater has one long elastic phase that lasts for 4 hours and other short ones that lasts for about 10 minutes. These occur approximately every 3 hours. The electric space heater with storage has one long elastic phase that lasts for about 4 hours. Its operation relies on external temperatures and the indoor temperature setpoint.

We have included an example of a load profile for a dishwasher that uses a local heating element for heating water obtained from [24] in Figure 1 to illustrate the typical power consumption of an appliance over time. The elastic phases that occur during the operating cycle of the dishwasher are also included in the diagram. Clearly, some of the profiles are deterministic in the default mode of operation (e.g., WM, TD, DW) and others can vary depending on external inputs (e.g., ESH with external temperature, EWH with hot water consumption).

From the discussion above, we have observed that every appliance has some combination of purely resistive, motor, electronic and storage components. We have identified appliances that have significant elastic phases. It is clear from the preceding discussion that the internal workings of appliances can vary from region to region and this can significantly affect the peak reduction capacity of the area. We are now ready to present the method used to select the reduced power consumption profile of elastic appliances.

\section{ASSUMPTIONS}

Our study is motivated by the presence of elastic components in many common household appliances. By reducing the power consumed by appliances currently in an elastic phase of their operation, instead of turning them completely off, we expect a significant reduction in residential peak demand with a much smaller impact on user comfort.

For the purpose of our study, we make three critical high- 
level assumptions. First, we assume that it is feasible to physically modify appliances to allow separate control of their elastic components and that appliance vendors are either motivated or compelled to do so. Second, we assume that heating components of appliances are well insulated. Therefore, if the power consumed by an elastic phase is reduced by a factor $\beta$, the time required to complete the phase increases in inverse proportion. Finally, we assume that when the power to an elastic phase is reduced, this does not affect the operation of inelastic components.

We also make the following technical assumptions. These are not critical to our scheme, but are necessary only to simplify analysis.

- We assume that the usage patterns of appliances are the same across all regions.

- We assume that there are three classes of appliances. Class 1 appliances operate once a day (e.g., the washing machine). Class 2 appliances operate in cycles over a day (e.g., the refrigerator). Finally, class 3 appliances operate more than once in a day but not cyclically (e.g. water heater).

- We assume that appliances belonging to class 2 operate continuously (worst-case use).

- We assume that households in all regions contain only the appliances discussed in Section 2.2, so that we can ignore the contributions of the less power-hungry appliances in our analysis.

- We assume that appliance load profiles used within a region are the same but can differ across regions.

- We assume that in all regions considered in this study, with the exception of India, electric space heaters are used only during winter ${ }^{1}$.

- Conversely, during the summer, we assume that air conditioners are used instead of space heaters (including in India).

- We assume that air conditioners are not used during winter in India.

- Since there is no penetration information for dryers or electric cookers in India, it is assumed that clothes are always air dried and alternate forms of cookers such as gas cookers are used.

- Lacking penetration information for ovens and hobs in, Ontario and Quebec, we assume that the penetration rate for these appliances in these provinces is the same as in France.

\section{APPROACH}

Given these assumptions, our approach is to propose a modified load profile for appliances with elastic components to minimize the peak power consumed during elastic phases while continuing to meet time and energy constraints. Specifically, the overall duration of operation of an appliance should not exceed the nominal one by a factor greater than $\alpha$ and

\footnotetext{
${ }^{1}$ Most regions in India are tropical and do not require the use of space heaters.
}

the overall energy consumed should be the same. We use an optimization framework (described in Section 4.2) to achieve this goal.

When appliances operate using this modified load profile, they will be referred to as operating in a "reduced mode". Otherwise, these appliances will be operating in "regular mode". The objective of our study is to compare how much peak reduction can be obtained when all elastic appliances use the reduced mode instead of the regular mode.

Before discussing our proposed method for modifying the load profile, we present the notation used in the remainder of this paper.

\subsection{Notation}

We consider the 10 appliances presented in Section 2.2. To identify the type of an appliance $j$, we define $k=\operatorname{AppType}(j)$ which returns a value from the set $\{W M, T D, D W, O, S$, $R F, F, A C, E W H, E S H\}$. The region in which an appliance $j$ resides in is $r=\operatorname{Region}(j)$ which returns a value from the set $\{O, Q, F, I\}$ which represents Ontario, Quebec, France and India. The fraction of homes in region $r$ containing an appliance of type $k$ is denoted as $\operatorname{pr}(k, r)$ and this is the penetration rate. We assume that each appliance has a "nominal" operating cycle used by all homes that have this type of appliance. This nominal operating cycle is assumed to be a succession of constant power phases (for example, referring to Figure 1, the nominal operating cycle of a DW has six phases, of which two are elastic).

Appliances of the same type from different regions can have different load profiles. Let the total number of elastic and inelastic phases in the nominal operating cycle of a type $k$ appliance in region $r$ be $m(k, r)$. Let the total duration of a nominal operating cycle for an appliance of type $k$ in region $r$ be $\Delta(k, r)$. Let the default duration of the $\ell^{t h}$ phase in the operating cycle of a type $k$ appliance in region $r$ be $\delta(\ell, k, r)$. The power consumed in the nominal mode by a type $k$ appliance in phase $\ell$ from region $r$ is $P(\ell, k, r)$. The time at which phase $\ell$ begins after the start of the operating cycle of a type $k$ appliance in region $r$ is denoted $t(\ell, k, r)$. Let $e(\ell, k, r)=1$ if phase $\ell$ in an appliance of type $k$ in region $r$ is elastic, otherwise $e(\ell, k, r)=0$.

The aggregate load in a neighborhood located in region $r$ with $N$ homes at time $t$ is denoted as $A(N, t, r)$. This is a random process representing the cumulative power drawn by all $N$ homes in region $r$ at a time instant $t$.

Let $\Delta(k, r)$ denote the nominal operating cycle duration of an appliance $k$ in region $r$ and let $\alpha$ denote the fractional increase in this duration. Then, we call the quantity $b(\alpha, k, r)=(\alpha-1) * \Delta(k, r)$ the delay budget that can be used to extend the duration of the appliance's elastic phases. There are many ways to allocate this budget across the elastic phases of an appliance $k$. We will now describe the one that we have selected, which results in the largest peak reduction of the three methods that we analyzed ${ }^{2}$.

\subsection{Power Reduction Method}

In our proposed method, when the operation duration of an appliance in region $r$ is extended by a factor $\alpha$, the power consumed during elastic phases is adjusted so that the maximum power consumed during any elastic phase is minimized according to the optimization problem $P_{0}(\alpha, r)$ stated below. Given $r$ and $\alpha$, the objective of our scheme is to minimize

\footnotetext{
${ }^{2}$ For reasons of space, we do not report the other two meth-
} ods here. 
the maximum power allocated to elastic phases so that time and energy constraints are met.

$$
\begin{array}{rr}
\mathbf{P}_{\mathbf{0}}(\alpha, r): \quad \min _{\left\{\delta^{\prime}(\ell, k, r)\right\},\left\{P^{\prime}(\ell, k, r)\right\}} X & \\
\text { s.t } \quad(\ell, k, r) P^{\prime}(\ell, k, r) \leq X, & \forall \ell, k \\
P(\ell, k, r) \geq P^{\prime}(l, k, r) \geq 0, & \forall \ell, k \\
\delta(\ell, k, r) \leq \delta^{\prime}(\ell, k, r), & \forall \ell, k \\
\alpha \Delta(k, r) \geq \sum_{\ell=1}^{m(k, r)} e(\ell, k, r) \frac{P(\ell, k, r)}{P^{\prime}(\ell, k, r)} \delta(l, k, r), & \forall k \\
\delta(\ell, k, r) P(\ell, k, r)=\delta^{\prime}(\ell, k, r) P^{\prime}(\ell, k, r), & \forall \ell, k
\end{array}
$$

where $P^{\prime}(\ell, k, r)$ is the modified power consumption of appliance $k$ in region $r$ during phase $l . \delta^{\prime}(\ell, k, r)$ is the new time required by appliance $k$ belonging to region $r$ to complete phase $\ell$. Equation 1d represents the time constraint set by $\alpha$. Equation 1e ensures that the energy consumed by the reduced mode remains the same as that consumed during the regular mode.

By replacing $P^{\prime}(\ell, k, r)$ by $\frac{\delta(\ell, k, r) P(\ell, k, r)}{\delta^{\prime}(\ell, k, r)}$ and $X$ by $\frac{1}{Y}$, the above optimization problem becomes the following linear optimization problem $P_{1}(\alpha, r)$ that can be easily solved.

$$
\begin{array}{rr}
\mathbf{P}_{\mathbf{1}}(\alpha, r): \max _{\left\{\delta^{\prime}(\ell, k, r)\right\}} Y & \\
\text { s.t } \quad e(\ell, k, r) P(\ell, k, r) \delta(\ell, k, r) Y \leq \delta^{\prime}(\ell, k, r), & \forall \ell, k \\
\delta(\ell, k, r) \leq \delta^{\prime}(\ell, k, r), & \forall \ell, k \\
\alpha \Delta(k, r) \geq \sum_{\ell=1}^{m(k, r)} e(\ell, k, r) \delta^{\prime}(\ell, k . r), & \forall k
\end{array}
$$

\section{EVALUATION METHODOLOGY}

This section evaluates the potential for peak demand reduction in a neighborhood of $N$ homes when all the appliances in the neighborhood operate in reduced mode using the optimization algorithm described in Section 4.2. We define peak reduction gain as follows. Suppose that the peak demand when appliances operate in the regular mode is $p L_{R}(r)$ and the peak load when appliances operate in the reduced mode is $p L_{M}(r)$, then the gain in peak reduction in $r$ is $\frac{p L_{R}(r)-p L_{M}(r)}{p L_{R}(r)}$.

We start by describing our data set in Section 5.1. This allows us to estimate an upper bound on peak load reduction, which we call the "peak demand reduction potential" of the region, using probabilistic computations (Section 5.2). The probabilistic method can become quickly intractable even for small $N$. We therefore resort to Monte Carlo simulations, as discussed in Section 5.3 for estimating the peak demand reduction potential for a larger value of $N$ that is more realistic. To confirm the accuracy of results from simulations, we show in Section 5.4 that the results obtained from simulations and computations are nearly identical for small values of $N$.

Note that the true peak demand of a neighborhood is not easily computed because of the presence of short-lived demand spikes. To eliminate the effect of these spikes, we pragmatically define the peak demand to be the largest aggregate power demand that occurs with a probability of at least $0.01 \%$.

\subsection{Data Set}

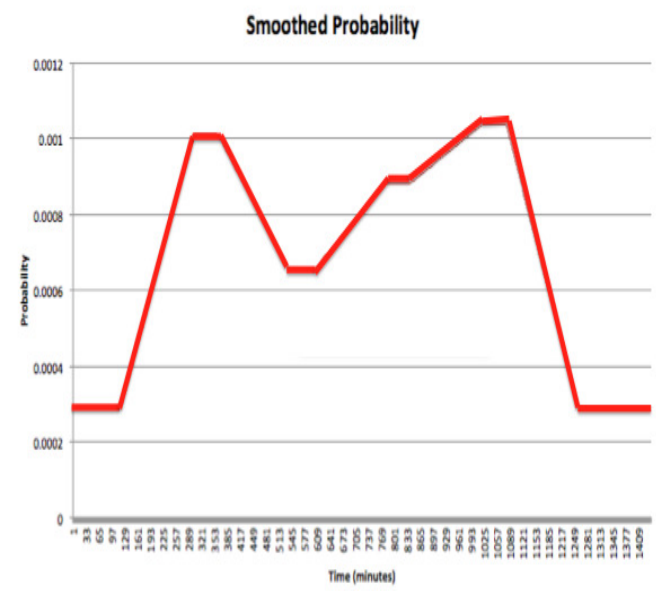

Figure 2: Distribution of a washing machine being on over a day.

The parameters used for all appliances in our computations and simulations are listed in Table 1 . The load profile of appliance $k$ is specified in Table 1 with the triple $(e(k, r), P(\ell, k, r), \delta(\ell, k, r))$ for every phase $\ell$. The load profiles and penetration rates of appliances in Ontario are obtained from [9], [17] and [2]. The data for Quebec is obtained from [17] and [3]. For France, data is obtained from [24]. Finally for India, data is obtained from [4] and [15].

From Table 1, it is clear that there are variations in the load profiles and penetration rates of appliances located in different regions. These differences lead to differences in the peak load reduction potential observed between regions. We now highlight the major differences observed in Table 1.

Note that washing machines and dishwashers in Ontario and India do not use a local heating element unlike those appliances in Quebec and France and hence these appliances are not elastic. The penetration rate of ACs in Ontario is more than twice the rates in the other regions considered in this study. Electric space heaters have a much higher presence in Quebec compared to any other regions.

Recall that we have assumed that appliances of the same type $k$, regardless of their region, have the same usage statistics. Reference [24] reports the frequency of operation of appliances over 5 intervals in a day, obtained from a survey of 2500 consumers in 10 European countries. We convert these statistics into a discrete probability distribution $\left(p_{o n}\left(k, t_{n}\right)\right)$ of a type $k$ appliance being "on" over a day at the scale of one minute by smoothing the discrete usage frequencies using a low-pass averaging filter. Figure 2 illustrates the resultant usage distribution of a washing machine.

Now that the data required to compute the peak reduction for all regions considered in our study is available, we can proceed to outlining how the peak reductions are obtained for a given region. The peak reduction is the difference between the peak demands when appliances operate in a regular mode and the peak demands when appliances operate in a reduced mode. In the regular mode each appliance follows its default operating cycle. In the reduced mode, we assume that an appliance of type $k$ in an elastic phase $\ell$ will draw a power computed according to $P_{1}(\alpha, r)$ so that the total duration of the operating cycle is now increased by a factor $\alpha$. 
We separately quantify the peak reductions for two seasons, winter and summer.

\subsection{Probabilistic Quantification}

One method for obtaining the probability distribution of the aggregate demand in a neighborhood belonging to region $r$ is through a probabilistic computation. As shown next, this computation is not tractable for large neighborhood with homes containing multiple appliances.

Consider a neighborhood in France where every home contains only one washing machine. A washing machine's operating cycle consists of $m(W M, F)=3$ phases, one of which is elastic. To simplify the derivation we introduce an "off" phase corresponding to the time when the appliance is not operating. The possible values that the aggregate load of a neighborhood of $N$ homes, each containing only a washing machine, can take is $C(N+4-1, N)$ (clearly some of these values can be equal). The probability, $p\left(W M, \ell, t_{n}, F\right)$, of a washing machine being in phase $\ell$ at discrete time $t_{n}$ can be computed as follows. The washing machine will be in phase $\ell$ at time $t_{n}$ only if it started its operation between $[L, U]$ where $L=t_{n}-t(\ell, W M, F)-\delta(\ell, W M, F)$ and $U=t_{n}-$ $t(\ell, W M, F)$. Hence, $p\left(W M, l, t_{n}, F\right)=\sum_{p=L}^{U} p_{\text {on }}(W M, p)$ (with a slight abuse of notation).

At time $t_{n}, i_{\ell}$ appliances are in phase $\ell$ for $\ell=1,2,3,4$ (note that $\sum_{\ell=1}^{4} i_{\ell}=N$ ), with probability of $\Pi_{\ell=1}^{4}\left(\begin{array}{c}N \\ i_{\ell}\end{array}\right) *$ $p\left(W M, \ell, t_{n}, F\right)^{i_{\ell}}$. The aggregate load in this case $A=$ $\sum_{\ell=1}^{4} i_{\ell} * P(\ell, W M, F)$. From this expression, we can derive the probability distribution of the aggregate load occurring over a day. This allows us to compute the probability that the aggregate load is greater than $x$ over a day.

However, using this method to compute the probability distribution of the aggregate load for a realistic neighborhood is intractable. For example, consider 100 homes, each containing only one appliance. Suppose this appliance has only 4 phases (including the "off" phase in which the appliance consumes $0 \mathrm{~W}$ ). Then there are 176,851 possible combinations to consider. In a realistic scenario, up to nine appliances (in a season either the AC or space heater cannot be used, hence the maximum appliances that can be used in a region per season is nine), each with different load profiles (and number of phases), may be active, making the computation combinatorially intractable.

\subsection{Monte Carlo Simulations}

This section describes an implementation of a Monte Carlo simulator to compute the probability distribution of the aggregate load when appliances are in regular or reduced mode. For every appliance in a neighborhood, start times are randomly selected according to the probability distribution of the appliance being active during the day. Appliances belonging to class 1 select this start time once over a day according to their usage probabilities. Appliances belonging to class 2 randomly select a start time in the "on" interval of the cycle every time a new cycle begins. Finally class 3 appliances randomly generate start times over a day according to their usage probabilities. The aggregate demand trajectory for a day is obtained by summing the load profiles of these appliances shifted according to their start times. When regular mode is being simulated, nominal load profiles are used. When reduced mode is simulated for a given $\alpha$, load profiles of appliances are modified according to $P_{1}(\alpha, r)$. We run the experiment for each scenario 1000 times for a given $N, \alpha$,

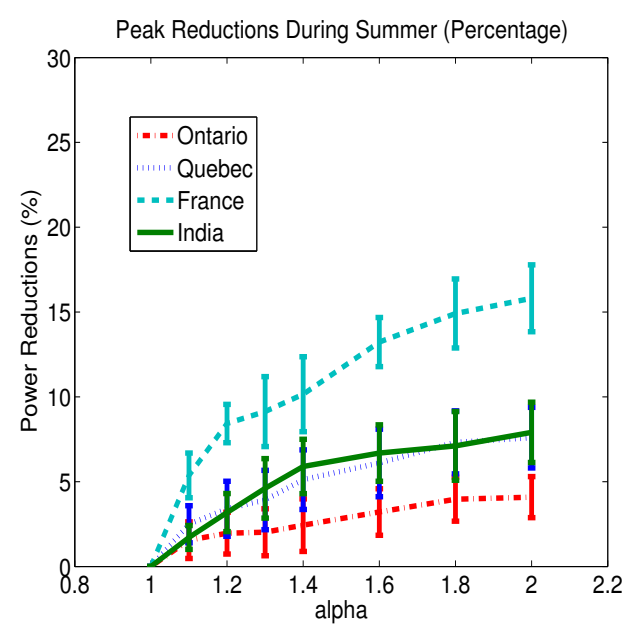

Figure 3: Comparison of percentage of peak reduction capacities during summer.

region and season. Then we present the averaged results.

\subsection{Comparison between Methods}

To validate the Monte Carlo implementation, we compare results from the probabilistic computation and the Monte Carlo simulation for $N=3$ homes located in France. It is assumed that these homes only contain a washing machine. For the computation, the derivation from Section 5.2 is used to obtain the probability distribution of all 20 possible aggregate loads for the neighborhood. The simulation is conducted as discussed in Section 5.3. The values obtained from the computation are treated as the "true" probability and compared with the $95 \%$ confidence interval around the mean probability value obtained from repeating the simulation 1000 times. We find that the probability obtained from the computation always lies within the $95 \%$ confidence intervals, and moreover, the width of the largest confidence interval is only $0.26 \%$ of the mean. We infer that, for this case, the two methods used to compute the probabilities are nearly identical, validating the Monte Carlo simulations. Therefore, in the remainder of the paper, we present results only from the Monte Carlo simulation.

\section{RESULTS}

We perform Monte Carlo simulations to obtain the peak reduction gain for neighborhoods in each region containing $N=100$ homes, each home containing appliances according to the penetration rates specified in Table 1. To understand the tradeoff between peak reduction gain and $\alpha$, the peak load reduction in each region is computed when $\alpha$ is varied from 1 to 2 . The results for summer and winter in all four regions are illustrated in Figure 3 and Figure 4.

Several interesting observations follow from Figure 3.

- France has the highest percentage of peak reductions during summer (about $15 \%$ for an $\alpha$ value of 2 . This is probably because this region has 6 major elastic appliances that are active during summer and a low penetration of (inelastic) ACs.

- India and Quebec have the next highest percentage of peak reductions during summer. Note that the peak 


\begin{tabular}{|c|c|c|c|c|}
\hline $\mathrm{k}$ & $\mathrm{r}$ & Class & $\operatorname{pr}(\mathrm{k}, \mathrm{r})(\%)$ & Nominal Load Profile (in tuples of $(e(k, r), P(\ell, k, r), \delta(\ell, k, r)))$ \\
\hline \multirow[t]{4}{*}{ WM } & Ontario & 1 & 85 & {$[(0,500 \mathrm{~W}, 30 \mathrm{~min})]$} \\
\hline & Quebec & 1 & 90 & {$[(1,2000 \mathrm{~W}, 15 \mathrm{~min}),(0,950 \mathrm{~W}, 15 \mathrm{~min}),(0,0 \mathrm{~W}, 30 \mathrm{~min}),(0,300 \mathrm{~W}, 15 \mathrm{~min})]$} \\
\hline & France & 1 & 95 & {$[\overline{(1,2000 \mathrm{~W}, 15 \mathrm{~min})},(0,0 \mathrm{~W}, 30 \mathrm{~min}),(0,300 \mathrm{~W}, 15 \mathrm{~min})]$} \\
\hline & India & 1 & 95 & {$[(0,700 \mathrm{~W}, 30 \mathrm{~min})]$} \\
\hline \multirow[t]{3}{*}{ TD } & Ontario & 1 & 35 & {$[(1,3000 \mathrm{~W}, 45 \mathrm{~min})]$} \\
\hline & Quebec & 1 & 29 & {$[(1,2200 \mathrm{~W}, 45 \mathrm{~min})]$} \\
\hline & France & 1 & 34 & {$[\overline{(1,2000 \mathrm{~W}, 45 \mathrm{~min})},(1,1550 \mathrm{~W}, 15 \mathrm{~min}),(1,1300 \mathrm{~W}, 15 \mathrm{~min}),(1,950 \mathrm{~W}, 15 \mathrm{~min})]$} \\
\hline \multirow[t]{4}{*}{ DW } & Ontario & 1 & 60 & {$[(0,50 \mathrm{~W}, 15 \mathrm{~min}),(0,500 \mathrm{~W}, 15 \mathrm{~min}),(0,250 \mathrm{~W}, 5 \mathrm{~min}),(0,125 \mathrm{~W}, 5 \mathrm{~min})]$} \\
\hline & Quebec & 1 & 55 & {$[(0,50 \mathrm{~W}, 15 \mathrm{~min}),(1,2000 \mathrm{~W}, 15 \mathrm{~min}),(0,50 \mathrm{~W}, 45 \mathrm{~min}),(1,2000 \mathrm{~W}, 15 \mathrm{~min})$,} \\
\hline & $\Gamma$ & & & $(0,250 \mathrm{~W}, 15 \mathrm{~min}),(0,125 \mathrm{~W}, 15 \mathrm{~min})]$ \\
\hline & France & 1 & 50 & $\begin{array}{l}{[(0,50 \mathrm{~W}, 15 \mathrm{~min}),(1,2000 \mathrm{~W}, 15 \mathrm{~min}),(0,50 \mathrm{~W}, 45 \mathrm{~min}), \underline{(1,2000 \mathrm{~W}, 15 \mathrm{~min})}} \\
(0,250 \mathrm{~W}, 15 \mathrm{~min}),\end{array}$ \\
\hline \multirow[t]{4}{*}{$\mathrm{RF}$} & Ontario & 2 & 100 & {$[(0,130 \mathrm{~W}, 15 \mathrm{~min}),(0,0 \mathrm{~W}, 15 \mathrm{~min})]$} \\
\hline & Quebec & 2 & 100 & {$[(0,130 \mathrm{~W}, 15 \mathrm{~min}),(0,0 \mathrm{~W}, 15 \mathrm{~min})]$} \\
\hline & France & 2 & 100 & {$[(0,138 \mathrm{~W}, 15 \mathrm{~min}),(0,0 \mathrm{~W}, 15 \mathrm{~min})]$} \\
\hline & India & 2 & 100 & {$[(0,200 \mathrm{~W}, 15 \mathrm{~min}),(0,0 \mathrm{~W}, 15 \mathrm{~min})]$} \\
\hline \multirow[t]{3}{*}{$\mathrm{F}$} & Ontario & 2 & 54 & {$[(0,130 \mathrm{~W}, 15 \mathrm{~min}),(0,0 \mathrm{~W}, 15 \mathrm{~min})]$} \\
\hline & Quebec & 2 & 51 & {$[(0,130 \mathrm{~W}, 15 \mathrm{~min}),(0,0 \mathrm{~W}, 15 \mathrm{~min})]$} \\
\hline & France & 2 & 52 & {$[(0,138 \mathrm{~W}, 15 \mathrm{~min}),(0,0 \mathrm{~W}, 15 \mathrm{~min})]$} \\
\hline \multirow[t]{4}{*}{$\mathrm{AC}$} & Ontario & 2 & 80 & {$[(0,2500 \mathrm{~W}, 15 \mathrm{~min}),(0,0 \mathrm{~W}, 15 \mathrm{~min})]$} \\
\hline & Quebec & 2 & 47 & {$[(0,2500 \mathrm{~W}, 15 \mathrm{~min}),(0,0 \mathrm{~W}, 15 \mathrm{~min})]$} \\
\hline & France & 2 & 12 & {$[(0,1700 \mathrm{~W}, 15 \mathrm{~min}),(0,0 \mathrm{~W}, 15 \mathrm{~min})]$} \\
\hline & India & 2 & 17 & {$[(0,1700 \mathrm{~W}, 15 \mathrm{~min}),(0,0 \mathrm{~W}, 15 \mathrm{~min})]$} \\
\hline $\mathrm{O}$ & France & 1 & 97 & {$[(1,600 \mathrm{~W}, 20 \mathrm{~min})$, repeat for 8 cycles: $(1,600 \mathrm{~W}, 3 \mathrm{~min}),(0,0 \mathrm{~W}, 2 \mathrm{~min})]$} \\
\hline S & France & 1 & 58.4 & $\begin{array}{l}{[(1,1500 \mathrm{~W}, 3 \mathrm{~min}),(0,0 \mathrm{~W}, 2 \mathrm{~min})} \\
\text { repeat for } 7 \text { cycles: } \\
(1,1500 \mathrm{~W}, 1 \mathrm{~min}),(0,0 \mathrm{~W}, 1 \mathrm{~min})]\end{array}$ \\
\hline \multirow[t]{4}{*}{ EWH } & Ontario & 3 & 23 & {$[(1,2000 \mathrm{~W}, 30 \mathrm{~min})]$} \\
\hline & Quebec & 3 & 23 & {$[\overline{(1,2000 \mathrm{~W}, 30 \mathrm{~min})}]$} \\
\hline & France & 3 & 45 & {$[\overline{(1,2000 \mathrm{~W}, 30 \mathrm{~min})}]$} \\
\hline & India & 3 & 30 & {$[(1,1500 \mathrm{~W}, 30 \mathrm{~min})]$} \\
\hline \multirow[t]{3}{*}{ ESH } & Ontario & 3 & 10 & {$[(1,8000 \mathrm{~W}, 2 \mathrm{~min})]$} \\
\hline & Quebec & 3 & 58 & {$[(1,8000 \mathrm{~W}, 2 \mathrm{~min})]$} \\
\hline & France & 3 & 22 & {$[\overline{(1,8000 \mathrm{~W}, 2 \mathrm{~min})}]$} \\
\hline
\end{tabular}

Table 1: Parameters used for each type of appliances (elastic phases are underlined).

demand in India is due to only four appliances. The percentage of peak reduction is computed with respect to this low value, which may account for the higher percentage of peak reductions for this region.

- Ontario has only a moderate peak reduction gain in summer (under $5 \%$ for an $\alpha$ value of 2). Ontario has high penetration rates for all appliances (especially ACs which are inelastic) and high absolute peak values, which accounts for the smaller fractional reduction in peak load.

From Figure 4, we see that, unlike the situation in the summer, the percentage of peak reductions is more or less the same for all four regions during winter.

We now consider, in more detail, the specific value of $\alpha=$ 1.1. This is of interest because a $10 \%$ increase in nominal operation time is likely to be barely noticeable by consumers. For example, the operation duration of a dryer in Ontario is 45 minutes. An extension by 1.1 of the operation duration of the appliance means that the total operation duration is extended to 49.5 minutes.

Table 2 shows the magnitude of peak reduction gain in

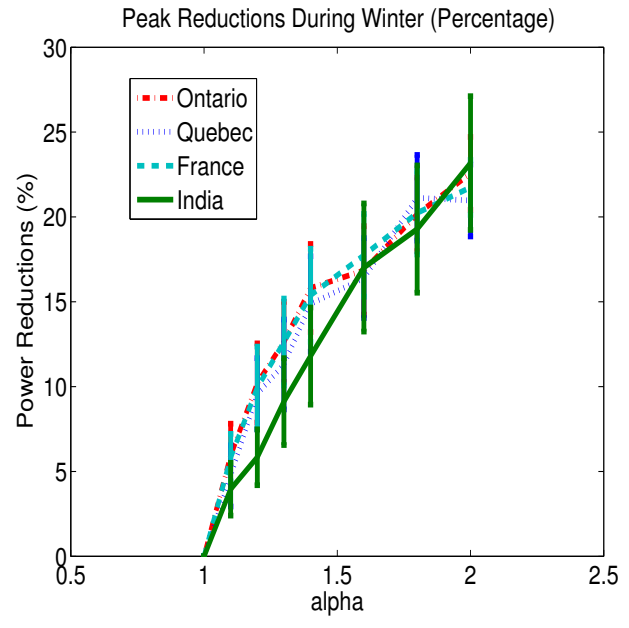

Figure 4: Comparison of percentage of peak reduction capacities during winter. 


\begin{tabular}{|c|c|c|}
\hline Region & Summer & Winter \\
\hline Ontario & $2200 \mathrm{~W}$ & $3500 \mathrm{~W}$ \\
Quebec & $3000 \mathrm{~W}$ & $3950 \mathrm{~W}$ \\
France & $3100 \mathrm{~W}$ & $3900 \mathrm{~W}$ \\
India & $200 \mathrm{~W}$ & $200 \mathrm{~W}$ \\
\hline
\end{tabular}

Table 2: Magnitude of Peak Demand Reduction for $\alpha=1.1$ in a neighborhood with $N=100$ homes.

every region for summer and winter for $\alpha=1.1$. We see that during summer both Quebec and France have the highest magnitude of absolute peak demand reductions. This is due to the higher proportion of elastic appliances in these regions: washing machines and dishwashers are elastic in Quebec and France unlike washing machines in Ontario and India. Similarly, during winter, both Quebec and France have a higher absolute magnitude of peak load reduction than Ontario and India. This can be attributed to the higher penetration rates of elastic appliances (e.g. washing machines, dishwasher, electric space heaters) in these regions. From the results presented in Figure 3, Figure 4 and Table 2, it is clear that the differences in the degree of peak demand reduction depend not only on the properties of appliances (which are different in every region), but also their differing penetration rates in each region.

Focusing now on Quebec (that has around 3.2 million households as of 2006 [7]) in winter, during which the peak period typically occurs [12], we see that, if all elastic appliances were using the reduced mode computed for an extension of $10 \%$ of the operation duration, we can expect a total residential peak rate reduction of about $125 \mathrm{MW}$ $(=3.2 * 3950 / 100)$. During summer, ACs are major contributors to peak demand. Since this reduction does not take into account the potential reduction in the peak load by careful scheduling of ACs [26], we see that a combined effort on AC and elastic household appliances could have a major impact on residential peak demand during summer.

\section{RELATED WORK}

Our work lies in the broad context of demand management schemes that reduce demand during conditions of peak load. These have been well studied in the research literature and are also widely used by utilities worldwide. In this section, we first give overview of the types of smart appliances that are available in the market today. Then, we discuss DR schemes that are currently in use by utilities for reducing demand overloads. Finally, we present a summary of DR schemes proposed in recent literature for residential load control.

\subsection{Smart Appliances}

Smart appliances are generally defined to be appliances that use computational intelligence to add operational flexibility. Surveys of traditional appliances can be found in References [9], [17], [3], [4] and [15].

Intelligence can be added to one of two types of appliances [14]. The first type have a fixed operation duration (e.g. washing machine, dishwasher). These typically operate once a day for a fixed period of time. The second set of appliances have thermal inertia and use energy to maintain a preset temperature (e.g. space heater, refrigerator). The first type of appliance can be made "smarter" by adding delay-start functions [14]. This allows consumers to postpone the start of the appliance according to time-of-use pricing.

This approach cannot be used for the second type of appliances because delaying the start time of operation for such appliances can cause the payback effect, which occurs when an appliance consumes more energy than needed when recovering from a postponement in start time. Hence, such appliances are made "smarter" by allowing consumers to define a range of acceptable temperature setpoints. When the need arises, the setpoint that requires the least work by the appliance is maintained [5].

The Smart-A project is a detailed study on smart appliances [24]. This report has compiled a comprehensive data set on load profiles of appliances and usage patterns of ten common domestic appliances in Europe. This report also makes recommendations on possible smart functions for each appliance covered in the report. None of these recommendations, however, leverage the elasticity property of appliances.

\subsection{Utility Demand Response (DR) Schemes}

Both price-based and incentive-based DR programs are currently in use by the utilities[21]: Price-based schemes bring humans into the control loop. The schemes that fall in this category include: Time-of-Use (TOU), Real-Time Pricing (RTP) and Critical Peak Pricing (CPP). All these schemes broadcast price signals to consumers who are expected to react appropriately (i.e. decrease their electricity consumption when the price increases). With TOU, the utility defines a price for a block of time - typically for a day. This price reflects the average cost to produce electricity. Consumers are aware of this pricing ahead of time and can plan their appliance usage accordingly. RTP is updated on an hourly basis and is based on actual generation costs. CPP is like TOU with the only difference being that during peak hours, a much higher price is set in place which reflects the actual generation costs [21]. RTP and CPP typically target large industrial loads. Price-based schemes depend entirely on human reaction to price signals and differ in the fixed time scales for which they are implemented.

Incentive-based schemes are contract-oriented. Utilities have a higher degree of control in these schemes. Schemes falling under this category include: Direct Load Control (DLC), Demand Bidding (DB) and Emergency DR (EDR). With DLC, the utility remotely shuts down power to heavy appliances such as ACs belonging to participating customers. Many studies have explored the use of DLC with ACs. The choice of appliances selected for the shut down is based on a solution to a complex optimization problem [26]. In the DB scheme, consumers place bids based on wholesale electricity market prices. The EDR scheme is tailored more for emergency or ancillary services. Customers commit to cutting down their loads upon receiving requests from the utility. Typically in these schemes, the utility provides incentives - mostly as monetary compensation to the participants. Customers can be penalized if they commit to a particular scheme and do not participate accordingly [21]. Incentive-based schemes are mostly geared to the industrial sector. Many such schemes have been abandoned at the pilot stages due to implementation complications.

Prior work in DR differs from our approach in many ways. To begin with, none of the preceding schemes decouple elastic and non-elastic components. Moreover, schemes such as 
DLC completely turn off appliances, which can affect the lifetime of sensitive non-elastic components. Existing DR schemes are centralized, do not take into account consumers' tolerance to comfort, and difficult to deploy. Finally, price signals used by schemes such as TOU are defined the day before: These signals do not reflect the instantaneous state of the grid.

When elasticity is used for DR, appliances operating their elastic phases can instantaneously respond to congestion signals received from the grid. The appliance operation extension factor is directly related to user comfort and this factor can be used to ensure that consumers are not too inconvenienced. If parameters such as appliance penetration, load profiles and usage patterns are available, then it even possible to estimate the peak demand reduction possible in that region, as we do in our study.

\subsection{Demand Response (DR) Schemes in the Research Literature}

We now discuss residential DR schemes that have been proposed in the recent literature. Until recently, DR schemes generally belonged to price-based and incentive-based categories as discussed in the previous section. Recently, many studies are making proposals for residential DR schemes that deviate from these two categories. In the following, we present an overview of such work.

References $[22,19,11]$ propose frameworks that schedule appliances in homes by constructing and solving optimization problems based on the day before TOU pricing information provided by the utilities.

In Reference [22], appliances in a home are classified into controllable and uncontrollable loads. Storage and renewable electricity generation are considered as part of a home energy management system. It is assumed that appliances operate at random times in a day, i.e. they do not follow a usage pattern. Controllable loads are time shifted when necessary. The load profiles of appliances are simple on-off models. Possible variations of power draw during the operation of an appliance is not considered. The notion of comfort is not considered in the scheduling problem.

Reference [19] uses particle swarm optimization to schedule controllable loads in a home. Numerical values to quantize benefits are assigned to energy services by consumers. Costs are reflected by the price of electricity based on TOU or CPP. The objective of the optimization problem is to maximize net benefits. This work heavily relies on the ability of consumers to assign a numerical value to the benefit of an energy service. It can be difficult for an consumer to quantify the benefits for energy services in a home. In our work, comfort is related to the duration required for an appliance to complete its operation. This is easier for consumers to interpret.

Reference [11], proposes a method to schedule appliances in between households in a neighborhood. This work divides appliances into two categories. In the first category, appliances must consume a fixed amount of energy and in the second category appliances can adjust power consumption with no constraints to the energy requirements. User satisfaction is incorporated for the second class of appliances in the form of utility functions. Our work defines elasticity at a finer granularity which is at the level of components instead of appliances as a whole. This is more realistic as our definition makes considerations as to how various components in the appliance can be affected due to variations in power drawn as will be described in Section 2.1. This paper uses an economic interpretation to solve the scheduling problem.

Reference [16] proposes a dynamic pricing scheme for utilities based on consumer benefits and grid conditions using utility functions. In this work, a vague notion of elasticity (in terms of power reduction and time shifting) is used. This concept is not formally defined in the paper. The modelling of load profiles of appliances in this paper is simplistic. The impact on various components in the appliances due to power changes is also not considered in this work.

Our work is different from these research efforts for the following reasons. First, we consider penetration rates, usage patterns and detailed load profiles to model the behaviour of appliances in a region instead of simplistic on-off load profiles in a single home. Second, we also consider possible adverse impacts on components due to variations in power supplied to them. Finally, we capture the notion of user comfort by the duration required by an appliance to complete its operation. We believe that this is easier to interpret by consumers.

\section{CONCLUSIONS}

We define the notion of appliance elasticity and exploit this property to define an optimization program that reduces appliance loads during demand peaks without overly affecting user comfort. We use comprehensive data sets to evaluate the potential benefits from our approach in four different regions around the world. Although our work makes several simplifying assumptions, we show a significant potential for reduction in peak demand due to appliance elasticity in all four regions considered in our study. This important because a reduction in the peak can cause:

- A reduction in the need to deploy new generation facilities, which can cost billions of dollars.

- A reduction in the carbon footprint of peak demands because carbon-intense generation facilities such as gas plants or coal plants are often commissioned to serve peak demands.

Given these potential reductions in peak load, appliance manufacturers should consider the design of appliances that, in addition to being energy efficient, also make an effort to reduce their peak power load. To achieve this goal, action from governmental regulators may be required, who can take one of two policy approaches. First, regulators could mandate appliance manufacturers to reduce peak power consumption of appliances with fixed appliance operation durations. The advantage of this approach is that, unlike a typical DR scheme, there is no need for communication between the utility and the consumer because appliances always operate at a reduced peak. However, this does not permit appliances to operate in regular mode when the grid is uncongested, which may frustrate consumers due to lengthier operation durations of appliances.

A second policy approach would be to require appliances to operate in a reduced mode during times of congestion. This permits appliance to operate in regular mode when the grid is uncongested, but requires infrastructure to transmit signals to appliances and appliance modification to respond to control signals. This policy is especially attractive in regions with deployment of smart meters with two-way communication capabilities. 
To understand whether there are significant gains from either policy, a detailed cost-benefit analysis is necessary for each scenario: we defer this to future work.

\section{REFERENCES}

[1] The Cornerstone of Energy Security in Quebec. http://hydroforthefuture.com/projets/19/, 2010.

[2] Appliance Calculator. http://www.hydroone.com/ MyHome/SaveEnergy/Tools/calc_main.htm, 2012.

[3] Consumption of Appliances. http://www.hydroquebec.com/energywise/ calculation-tools/index.html, 2012.

[4] Monthly energy consumption calculator. http://www.pgvcl.com/calculate.htm, 2012.

[5] D. Angeli and P. A. Kountouriotis. Decentralized Ransdom Control of Refrigerator Appliances. IFAC World Congress, 2011.

[6] E. Bonneville and A. Rialhe. Demand Side Management for Residential and Commercial End-Users, 2006.

[7] S. Canada. Housing and Dwelling Characteristics. http://www40.statcan.ca/101/cst01/famil55b-eng.htm, 2006.

[8] Central Electrical Authority. Report on 17th Electric Power Survey in India, 2007.

[9] J. Elder. Power Consumption of Some Household Appliances. http: //web.ncf.ca/jim/misc/killawatt/index.html, October 2006.

[10] Energy Information Administration. U.S. Household Electricity Report. http://www.eia.doe.gov/emeu/ reps/enduse/er01_us.html, 2005.

[11] N. Gatsis and G. B. Giannikis. Cooperative Multi-Residence Demand Response Scheduling. In IEEE 45th Annual Conference on Information Sciences and Systems, 2011.

[12] Hydro Quebec. Annual Report 2010. "http://www.hydroquebec.com/publications/en/ annual_report/pdf/rapport-annuel-2010.pdf".

[13] A. Ipackchi and F. Albuyeh. Grid of the Future. IEEE Power and Energy Magazine, 7:52 - 62, 2009.

[14] T. Jamasb and M. Pollitt. The Future of Electricity Demand: Customers, Citizens and Loads. Cambridge University Press, 2011.

[15] V. Letschert and M. A. McNeil. Coping with Residential Electricity Demand in India's Future How Much Can Efficiency Achieve?, 2007.

[16] N. Li, L. Chen, and S. T. Low. Optimal Demand Response Based on Utility Maximization in Power Networks. In IEEE Power and Energy Society, 2011.

[17] Natural Resources of Canada. Survey of Household Energy Usage, 2007.

[18] Ontario's Power Authority. Ontario's Long Term Energy Plan, 2010.

[19] M. A. A. Pedrasa, T. D. Spooner, and I. F. MacGill. Coordinated Scheduling of Residential Distributed Energy Resources to Optimize Smart Home Energy Services. IEEE Transactions on Smart Grid, 1:134 143, 2010.

[20] G. Pistoia. Electric and Hybrid Vehicles: Power Sources, Models, Sustainability, Infrastructure and the
Market. Elsevier, Jordan Hill, Oxford, 2010.

[21] Report to the United States Congress. Benefits of Demand Response in Electricity Markets and Recommendations for Achieving them, 2006.

[22] C. Roe, S. Melipoulos, R. Entriken, and S. Chhaya. Simulated Demand Response of a Residential Energy Management System. In IEEE Energy Tech, 2011.

[23] A. Shalaby. Ontario's Integrated Power System Plan: Load Forecast, 2006.

[24] R. Stamminger. Smart-A Project: Synergy Potential of Smart Appliances, 2008.

[25] A. Von Meier. Electric Power Systems: A Conceptual Introduction. John Wiley and Sons Inc., New Jersey, 2006.

[26] D. Wei and N. Chen. Air Conditioner Direct Load Control by Multi-Pass Dynamic Programming. IEEE Transactions on Power Systems, 10:307-313, 1995. 